\title{
La ciencia bibliotecológica y de la información ¿tradición o innovación en su paradigma científico?
}

\author{
Miguel Ángel Rendón Rojas \\ Centro Universitario de Investigaciones Bibliotecológicas \\ de la UNAM, 04510, México D.F., Tel: (525)623-03-41 \\ E-mail: marr@ servidor.unam.mx
}

\section{RESUMEN}

El desarrollo de la teoría sobre la información documental plantea el dilema de o continuar en el mismo paradigma seguido porla tradición bibliotecológica o bien cambiara uno nuevo. Después de un análisis crítico sobre las ideas del desarrollo científico de T. Kuhn eI. Lakatos, se concluye que la elección no es excluyente. Empleando la terminología de Lakatos, pero haciéndole algunas aportaciones a sus propuestas, concluimos que la ciencia bibliotecológica y de la información conserva elementos de la tradición dentro de lo que llamamos su "núcleo duro" depurado, y a la vez presenta elementos de innovación en el "cinturón protector", el cual incluye una serie de teorías, emanadas del núcleo central, queestán encargadas de estudiar las nuevas realidades.

Palabras clave: Tería de la Bibliotecología; Desarrollo de la Bibliotecología; Thomas S. Kuhn; Imre Lakatos.

\section{THE SCIENCE OF LIBRARY SCIENCE AND INFORMATION: A PARADIGMOF TRADITION OR OF INNOVATION? Miguel ÁNGEL RENDóN-RojaS}

\section{ABST RACT}

The development of a theory on documental information posits the dilemma of either continuing with thetime-honored paradigm followed by Library Science or rather shifting to a new one. After a critical analysis of the scientific ideas of T. Kuhn and I. Lakatos, the study concludes that the dilemma is false. Using Lakatos' terminology, and offering some addenda to his proposals, we conclude that the science of Library Science and Information continues to exhibit traditional elements in what we call a refined "hard nucleus," while at the same time exhibiting innovative characteristics in a sort of "protective belt," which includes a series of theories, emanating from the nucleus, that are attempts to grasp the new realities.

Key Words: Librarianship Theory; Librarianship Development; Thomas S. Kuhn; Imre Lakatos.

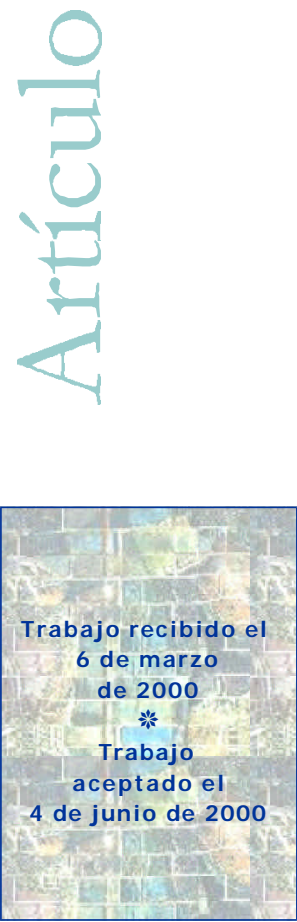




\section{LA CIENCIA BIBLIOTECOLÓGICA Y DE LA INFORMACIÓN ANTE EL DILEMA DE TRADICIÓN O INNOVACIÓN}

T l ser se va manifestando al hombre en formas concretas y tomando distintas "apariencias" según la etapa histórica en que se muestra. El estudio referenteal mundo de la información documental y fenómenos relacionados con él, había "aparecido" tradicionalmente como el estudio de un campo debidamente identificado, como lo es el mundo de los libros, de las bibliotecas y de los bibliotecarios, ${ }^{1}$ donde el quehacer para trabajar con ese mundo se traducía en actividades para conservarlo y, gracias a su análisis, organización y sistematización, ${ }^{2}$ cumplir con el ideal derivado de la Ilustración de proporcionarle servicio público a usuarios. Con el tiempo el mundo de los libros y de los impresos fue quedándose reducido, por lo que una primera transformación implicó aceptar dentro de ese campo mencionado a una clase más amplia de documentos que incluía no solamente a los impresos sino a cualquier registro, sin importar el tipo de soporte, y a otras unidades de información documental aparte delas bibliotecas. Sin embargo , esa "concesión" no fue suficiente y la realidad desbordó a la visión tradicional: los avances tecnológicos, las ascendentes expectativas de los usuarios y factores de índole económica, ${ }^{3}$ a los que también agregaríamos los procesos de especialización de la ciencia y la interdisciplinariedad; la globalización de la sociedad, la dinámica del cambio en todas las esferas de la vida contemporánea, donde el flujo de información influye directamente en esos cambios y a su vez es influido por ellos, y la desmaterialización (virtualidad) de los fenómenos antes tan bien conocidos (documentos, unidades de información, usuarios) todo lo cual motivó el replanteamiento dela visión tradicional y que se empezara a hablar de fenómenos más abstractos, tales como la información o el conocimiento, como un nuevo objeto de estudio deuna nueva disciplina o como un viejo nuevo objeto de estudio de una antigua disciplina.

En el primer caso, cuando se reconoce la aparición de una nueva ciencia, la propuesta es que ese lugar sea ocupado por la ciencia de lainformación, la cual según $\mathrm{H}$. Borko es "una ciencia interdisciplinaria que investiga las propiedades y comportamiento de la información, las fuerzas que gobiernan el flujo y el uso de la información y las técnicas, manuales y mecánicas, del proceso informativo para el óptimo

1 De ahí la raíz bibliode los términos biblioteconomía, bibliotecología, bibliología, bibliografía.

2 Aunque en ocasiones se da más énfasis a la conservación, como lo podemos constatar en Shera: "[...] todas las funciones de la biblioteca convergen en la conservación [...] la conservación es básica y fundamental para la biblioteca." J. Shera. Los fundamentos dela educadón biblictedoógica México: UNAM/ CUIB, 1990. p. 142.

3 R. D avies. "O utlines of the emerging paradigm cataloguing". En Information, ProcessingandManage ment. V. 23. N. 2. p. 90. 
almacenamiento, recuperación y diseminación" ;4 o bien por las ciencias de la información, a las que R. Taylor define como "1) El estudio de las propiedades, estructura y transmisión de conocimiento especializado y 2) El desarrollo de métodos para su útil organización y diseminación." 50 bien se propone que el lugar lo ocupen la documentación, que es la "ciencia general que tiene por objeto el estudio del proceso de adecuación y transmisión de las fuentes para la obtención de nuevo conocimiento"; 6 o la documentología que es la "ciencia y práctica de la elaboración y la organización de la información en todos los dominios científicos y técnicos, comprendidos los de la economía y la sociología"; 7 o la Informatología; ${ }^{8}$ o la Informatika, que Mijáilov y coautores definen como la "disciplina que estudiala estructura y las propiedades (y no el contenido específico) de la información científica, así como las leyes que rigen la actividad científico-informativa, su teoría, historia, metodología y organización. [Cuyo objetivo es] elaborar métodos y medios óptimos de presentación (registro), recolección, procesamiento analítico-sintético, almacenamiento, búsqueda y diseminación de la información científica." 90 también podría ocupar ese lugar la Ingeniería de la Información, que tiene por objeto "el desarrollo, diseño y operación de los sistemas de información, que incluyen bibliotecas, servicios de indización y resúmenes, y centros de información y de datos"; 10 o la Ingeniería del Conocimiento que según $\mathrm{H}$. Schildt es la "disciplina que trata de la forma en que se organizan, construyen y verifican las bases de conocimientos."11

En el segundo caso, un viejo objeto de estudio de una antigua disciplina, no se acepta la aparición de una nueva ciencia sino que se reconoce la continuidad de la bibliotecología, aunque se precisa su nuevo campo de estudio. D e esta manera, se define a la bibliotecología como "la ciencia que estudia: el registro y flujo del conocimiento y

4 H. Borko. "Information Science. What is it?". En American Doamentation Vol. 19. N. 1, enero 1968. pp. 3-5. p. 5.0 tro concepto es que la Ciencia de la Información es "el cuerpo de conocimiento compuesto de descripciones, teorías y técnicas, que proporciona la comprensión de los medios por los que las necesidades de información de la sociedad son satisfechas y las competencias para definir y satisfacer tales necesidades". Hosovsky; Alexander G. y Massey, Robert J. "Information Sciences: Its ends, means and opportunities". En Procedings of theASIS. Vol. 5, 1968. pp. 47-55. p. 47. La bibliografía so bre Ciencia de la información es muy grande, una revisión general de este concepto se encuentra en López Y epes, José. La doamentacón comodisaplina. Teańa ehistaria España: EUNSA, 1995. Cap. VI.

5 Robert S. Taylor. “The Information Sciences”. En LibraryJaumal. Nov. 1963. pp. 4161-4163. p. 4161.

6 José López Y epes. Op Cit p. 322.

7 D. Isakovic. "La documentologie". En ReveIntemationaledelaDoumentation Vol. 32, fasc. 4, 1965. pp. 152-153.

8 Pauline Altherton. "Letter to Editor". En American Doumentation Vol 16. N. 2, 1965. P. 126.

9 A. I. Mijáilov; A. I. Chernii; y R. S. Gilyarevskii. Fundamentos dela Infomática Moscú-La Habana: Nauka; A cademia de Ciencias de Cuba; Instituto de D ocumentación en Información Científica y Técnica. 1973. V. 1. p. 57.

10 Robert S. Taylor. "Professional Aspects of Information Science and Technology". En Amual Re viewof Information ScienceandTechndogy.V . 1. 1966. pp. 15-39. p. 17.

11 Herbert Schildt. "Turbo Prolog programación avanzada. México: MacG raw-Hill, 1990. P. 87. 
de la información; así como la circulación social de los medios que la contienen para hacer posible su uso y organización." 12

Todo esto hizo surgir la inquietud de realizarinvestigaciones metabibliotecológicas que nos permitieran vislumbraryjustificar el alcance y dirección de esos cambios teóricos en la disciplina. La pregunta por responder era ¿hay que adoptar una posición teórica totalmente nueva o refugiarse en la tradición ya probada? Es así como aparece el dilema ante los especialistas de la información documental: o tradición 0 innovación, o evolución o revolución científica.

\section{T. KUHN Y LAS REV OLUCIONES CIENTÍFICAS}

D ebido a que el planteamiento de T. Kuhn sobre el desarrollo de la ciencia hace referencia a periodos de crisis y de revoluciones científicas, situación que muy bien se podía aplicar al estudio de la información documental (vista como ya señalamos, como un proceso evolutivo o revolucionario), y a que un término central en el pensamiento de Kuhn es lo que llamó en un principio paradigma, se empezó a emplear el término kuhniano de paradigma y cambio de paradigma; ${ }^{13}$ por lo que el dilema mencionado anteriormente puede ser expresado en términos de continuar con el mismo paradigma científico; es decir, aceptar la tradición y rechazar la innovación, o bien adoptar uno nuevo, y por lo tanto romper con la tradición y lanzarse en brazos de la innovación.

Por desgracia el mismo Kuhn, en la Posdatade1969reconoce que en su "texto original no deja ninguna cuestión más oscura o más importante" 14 que la de paradigma. Y al intentar clarificarlo, en ese mismo escrito, distingue dos sentidos de paradigma: como logro o solución de un problema reconocido por la comunidad, y como "constelación de compromisos de grupo." A este último, que es el sentido de paradigma como marco de investigación y que es el que más se ha utilizado, "para evitar confusiones" sugiere llamarlo "matriz disciplinaria."15

12 Ramiro Lafuente López y Estela Morales Campos. "Reflexiones en torno a la enseñanza de la bibliotecología”. En Investigadón biblictedoóġa: archivonomáa, biblideedoǵa e informacón México: UNAM/ CUIB. Vol. 6. N. 12. pp. 25-33. p. 25.

13 Cfr. Ramiro Lafuente López. “¿Es necesario un nuevo paradigma en catalogación? En Investigacón biblicteedógica: archivonomáa, biblictedoǵa einfomación México: UNAM/ CUIB. Vol. 3. N. 5. pp.4-12. Solange Puntel Mostafa. "Enfoques paradigmáticos da bibliotecologia; unidade na diversidad ou diversidad na unidad”. En Investigacónbiblideedógica: archivonomá, biblideedoǵaeinfomadón México: UNAM/ CUIB. Vol.10. N. 21.pp. 18-21. Incluso nosotros adoptamos esa terminología. Cfr. Miguel Ángel Rendón Rojas. "Hacia un nuevo paradigma en Bibliotecología”. En Transinfomacãa S. P. Brasil: PUCCAMP. V. 8. N. 3. pp. 17-31; “Comentario. X IV Coloquio de Bibliotecología. Los diferentes enfoques paradigmáticos de la ciencia bibliotecológica: unidad en la diversidad o diversidad en la unidad". En Investigacoón biblictedógica: ardivonamá, biblidtedoóa e informacón México: UNAM/ CUIB. Vol. 10. N. 21. pp.2-3.

14 Thomas Kuhn. Posdata: 1969. En La estructura delasrevdudionesdientíficas México: FCE, 1996. D uodécima reimpresión. p. 278.

15 Ibid p. 279. 
D entro de la matriz disciplinaria, existen, según Kuhn, varios componentes, de los cuales analiza cuatro. El primero, que denomina "generalizaciones simbólicas", "parecen leyes de la naturaleza, pero para los miembros del grupo, su función a menudo no es tan sólo esa [...] funcionan en parte como leyes, pero también en parte como definiciones de algunos de los símbolos que muestran."16 Según parece, este componente proporciona la posibilidad de compartir un "juego de lenguaje" entre la comunidad, lo que ofrece la posibilidad de tener una terminología, semánticay un uso común del lenguaje. El problema en este componente del paradigma tradicional de la bibliotecología se manifiesta en la ausencia y consecuente búsqueda de términos y conceptos comunes, tales como documento electrónico, biblioteca virtual y metadato, entre otros. Un ejemplo de la necesidad de encontrar un nuevo consenso en este elemento paradigmático son los trabajos que últimamente se han realizado en terminología, vocabularios especializados y tesauros. ${ }^{17}$

El segundo componente de la matriz disciplinaria son los compromisos ontológicos compartidos, creencias en modelos particulares que pueden incluir la convicción de que existen ciertos objetos y procesos, o la aceptación de ciertas analogías 0 metáforas. Esto ayuda a establecer "lo que será aceptado como explicación y como solución de problemas [...] en la determinación de la lista de enigmas no resueltos y en la evaluación de la importancia de cada uno." 18 Este componente ofrece la posibilidad de compartir un "mundo" que merece ser estudiado y que al mismo tiempo ofrece soluciones para los problemas que plantea. Un ejemplo claro de reestructuración del "mundo bibliotecológico" es la reestructuración delas áreas de investigación del CUIB/ UNAM y la discusión sobre la necesidad de la existencia o no del área de tecnologías de la información; o la identificación de un nuevo fenómeno digno de ser estudiado desde la perspectiva bibliotecológica, como es la sociedad de la información o del conocimiento.

El tercer elemento de la matriz disciplinaria son los valores compartidos por la comunidad epistémica. Los valores mencionados por Kuhn son los que serefieren a la amplitud y precisión en las predicciones, a la fecundidad para formular y solucionar enigmas, a la sencillez teórica, y a su coherencia interna y externa con otras disciplinas. Este componente orienta la intencionalidad (direccionalidad) de la actividad investigadora. Una inquisición motivada por el sentimiento deinsatisfacción en este componente paradigmático de la bibliotecología son los trabajos que versan sobreel problema de la metodología de dicha disciplina. ${ }^{19}$

16 Ibid p. 281.

17 Cfr. Araceli Torres Vargas. Linææmientos para \& análisis detéminos en Biblictedoǵa UNAM/ CUIB, 1998 y Teaaurolatimoammicamoen denaiabiblictedóǵca ydela informaión Catalina Naumis Peña; $\notin a l$. México: UNAM/ CUIB, 1999.

18 Thomas Kuhn. Op Cit p. 283.

19 Crr. Valentino Morales López. Aproximadionesal conceptodeMetoddoǵa enlaBiblictedoǵa Tesis para obtener el G rado de Maestro en Ciencias con especialidad en Metodología de la Ciencia. México, D. F. I.P.N/ PESTYC, 1999. 
El cuarto componente, aunque no es el último que resta, se refiere a los ejemplos paradigmáticos, "las concretas soluciones de problemas que los estudiantes encuentran desde el principio de su educación científica [...] y que les enseñan, mediante el ejemplo, cómo deben realizar su tarea. "20 La problemática en este elemento de la ma triz disciplinaria de la bibliotecología la muestran las discusiones en los cambios de los planes y programas de estudio en las diferentes licenciaturas de bibliotecología en las universidades del país que las ofrecen. Incluso en el caso del Colegio de Bibliotecología de laUNAM, la discusión continúa porqueno se encuentraun modelo satisfactorio que sirva de base para que las nuevas generaciones "aprendan" bibliotecología.

Con base en ese concepto central de paradigma o matriz disciplinaria, Kuhn afirma que las diversas disciplinas científicas se desarrollan de acuerdo con una ctructura geneal que comienza con una etapa "precientífica" (preparadigmática) en la que "excluyendo los campos, tales como las matemáticas y la astronomía, en los que los primeros paradigmas firmes datan de la prehistoria, y también los que, como la bioquímica, surgieron por la división o la combinación de especialidades ya maduras", ${ }^{21}$ no existía una opinión única sino la competencia entre diversas escuelas sobre lo que se debía investigar (compromiso ontológico), sobre cuáles eran los conceptos centrales (generalizaciones simbólicas), cuáles las respuestas correctas (ejemplos paradigmáticos) y los instrumentos técnicos y metodológicos que se debían emplear, cuáles las interpretaciones "canónicas", etcétera. Este periodo termina cuando los distintos investigadores aceptan un mismo marco de principios básicos para hacer su investigación (paradigma) y se integran ellos mismos como comunidad científica y se reconocen como tal, tras lo cual aparece el periodo de "ciencia normal". D urante esta etapa la ciencia normal se dedica a resolver enigmas, esto es, a resolver problemas científicos, pero siguiendo las "reglas" establecidas por el paradigma; es decir, se reconocen los problemas, se recolectan datos, y se interpretan y analizan de acuerdo con el modelo establecido por el paradigma. El marco de principios básicos se acepta y no se cuestiona ni se considera problemático o sujeto a revisión. Se trabaja todo el tiempo con las mismas reglas de juego y esto permite que se dé la acumulación de conocimientos. El paradigma es una promesa para ampliar exitosamente el conocimiento resolviendo enigmas, y si existen fracasos, la culpa es de la poca capacidad de los investigadores y no del marco de principios aceptado. Sin embargo siempre llega un momento en que la ciencianormal, debido a su amplitud explicativa y al aumento de su contenido informativo (cuanto más se diga, más riesgo hay de equivocarse; quien dice nada, nunca se equivoca; si se dice poco, hay poco riesgo de cometer errores) se enfrenta a un problema que no puede resolver no por falta de habilidad del científico, sino porque en cierta forma "la naturaleza ha violado las expectativas, inducidas por el paradigma, que rigen a la ciencia normal” 22 y se resiste

20 Ilid p. 286.

21 T. Khun. Op Cit. p. 40.

22 Ibid p. 93. 
a ser resuelto con las herramientas instrumentales y teóricas del paradigma. Ese fenómeno no debería existir, no tiene concepto ni explicación teórica. Es así como comienza la etapa de "crisis" y con ella la ciencia extraordinaria. En esta etapa los investigadores tienen la oportunidad de ensayarlo todo. "Los primeros intentos de resolución del problema seguirán de cerca las reglas establecidas por el paradigma; pero al continuar adelante sin poder vencer la resistencia, las tentativas de resolución involucrarán, cada vez más, alguna coyuntura menor o no tan ligera del paradigma [...] las reglas de la ciencia normal se hacen cada vez más confusas" .23 D e esta manera, "cada vez más, la investigación [se va] pareciendo a la llevada a cabo por las escuelas en competencia del perio do anterior al paradigma" . 24 Finalmentela crisis se termina cuando el paradigma que fue puesto en tela de juicio se muestra capaz de resolver las anomalías o surge un paradigma alternativo que parece ofrecer una respuesta a la anomalía, además de que promete guiar la investigación por un camino fructífero de soluciones de enigmas. Comienza así la lucha por lograr un nuevo consenso hasta que el paradigmaalternativo vence y es aceptado por la comunidad científica, tras lo cual puede decirse que ha tomado lugar una revolución científica.

El anterior desarrollo de las ciencias puede ser representado esquemáticamente de la siguiente manera:

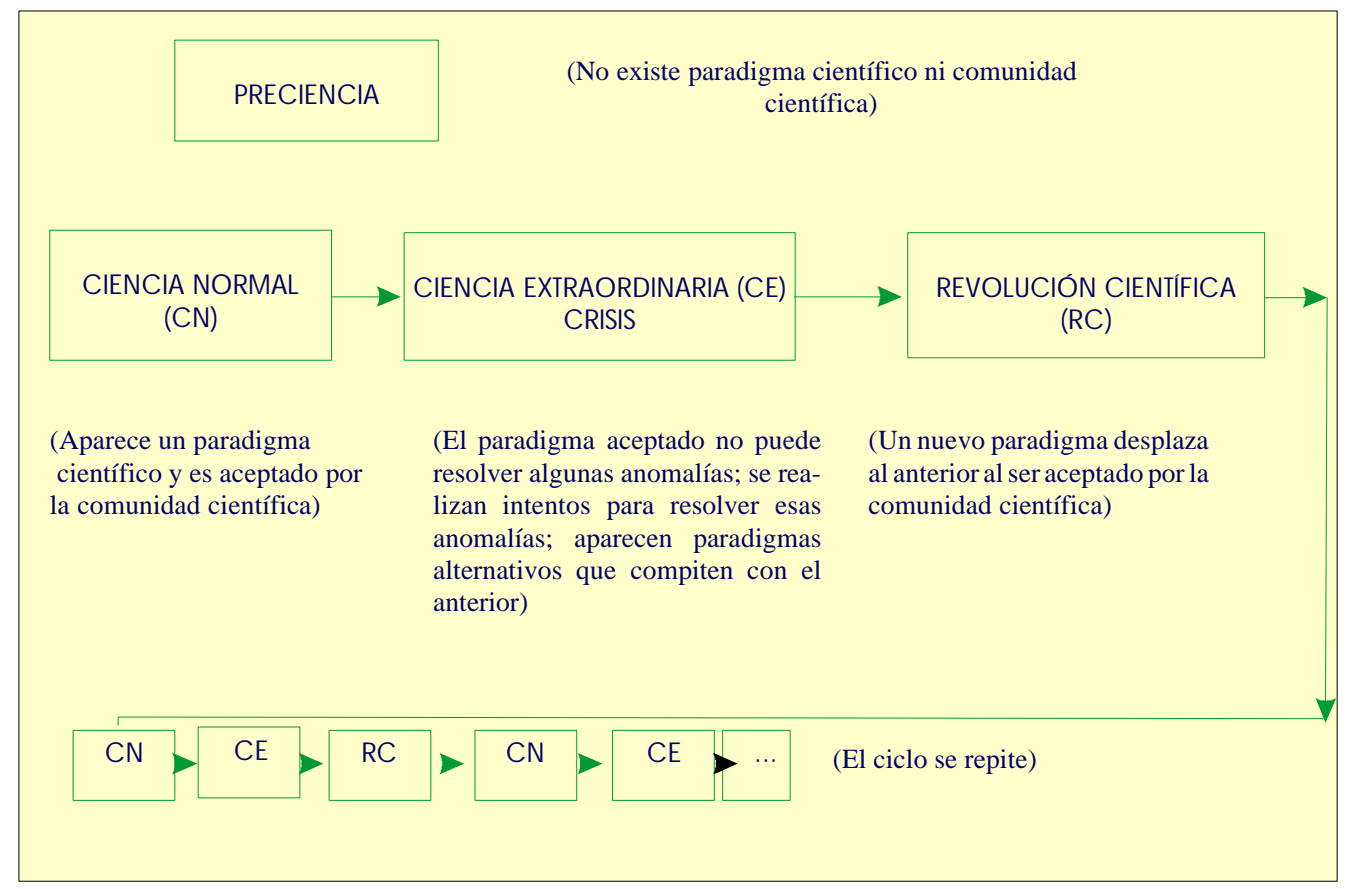

23 Ibid p. 136-137.

24 Ilid p. 121. 
La visión kuhniana del desarrollo científico es atractiva porque toma en cuenta factores que otros han dejado pasar, como el papel comunitario de la actividad científica y, sobre todo, su historicidad, avalada por los muchos ejemplos que nos proporciona la historia de la ciencia. En el ambiente bibliotecológico, como anotamos al describir los componentes de la matriz disciplinaria o paradigma, se percibe la existencia de elementos suficientes para afirmar que el modelo de Kuhn sobre la revolución científica trabaja correctamente en el campo de labibliotecología y permite comprender el desarrollo de la disciplina. 25

Sin embargo, según nuestra opinión, el modelo kuhniano deja un sentimiento de insatisfacción al concebir el desarrollo de la ciencia como una serie de rupturas entre los paradigmas, como grandes saltos que se producen al cambiar de una forma de hacer ciencia a otra, sin que se puedan homologar completamente ni decidir cuál de estas ciencias es "más verdadera", porque los nuevos conocimientos no reemplazan a la ignorancia sino a otros conocimientos de tipo distinto e incompatible. ${ }^{26}$ Esas rupturas y saltos se deben a lo que Kuhn denominó inconmensurabilidad entre paradigmas, la cual es el resultado de un constructivismo que, aunque no tan radical como el del idealismo subjetivo de Berkeley (en el que el sujeto crea, en el sentido ontológico, la realidad) sí es el de un subjetivismo de tipo kantiano, porque si bien se reconoce la existencia de un mundo dado que no varía pese a los cambios de paradigmas, éste permanece en el nivel obscuro y desconocido de "la cosa en sí", y sólo puede ser "visto" a través del paradigma, el cual le da forma (Gestalt). Por consiguiente, si cambia el paradigma, cambia la forma de verlo. ${ }^{27}$

D e esta manera, la inconmensurabilidad nos conduce a un solipsismo colectivo porque plantea un abismo entre los mundos de comunidades científicas que tienen diferentes paradigmas, debido a que "quienes proponen los paradigmas en competencia practican sus profesiones en mundos diferentes". ${ }^{28}$ Asimismo la inconmensurabilidad determina una incapacidad de comunicación entre las distintas comunidades paradigmáticas, puesto que aunque los nuevos paradigmas "incorporan ordinariamente gran parte del vocabulario y de los aparatos, tanto conceptuales como de manipulación [...] es raro que empleen exactamente del modo tradicional a

25 Por supuesto la aplicación del modelo kuhniano al desarrollo de la bibliotecología se hace con todas las reservas del caso, puesto que el mismo Kuhn expresó que las ciencias sociales y la bibliotecología, a la que sitúo dentro de las ciencias del espíritu (humanas y sociales), se encuentran en la etapa pre-paradigmática. Pero concedemos que todos los siglos de desarrollo práctico y el último siglo aproximadamente de desarrollo teórico de la bibliotecología rindieron sus frutos para conformar una comunidad epistémica que se reconoce a sí misma por compartir ciertos presupuestos iniciales. D ichos presupuestos se pueden identificar como lo que hemos llamado "paradigma tradicional." Cfr. T. Kuhn, Op Cit. p. 40.

26 Ibid p. 154.

27 Por supuesto que el paradigma que actúa como elemento de síntesis en la forma de construir el mundo para la comunidad científica no es una forma a prioi de conocimiento.

28 Ibid p. 233. 
esos elementos que han tomado prestados [por lo que] el resultado inevitable es lo que debemos llamar [...] un malentendido entre las dos escuelas en competencia." 29

Sin embargo cabe señalar que en otros escritos Kuhn reconoce la existencia de elementos comunes entre distintos paradigmas, ya que inconmensurabilidad no significa intraducibiblidad total o incomprensibilidad, sino únicamente que dos teorías están articuladas en lenguajes queno son completamente traducibles entre sí, lo que permite cierta traducibiblidad, aunque no "punto por punto". A pesar de que no puede darse una traducción completa, se puede llegar a la "comprensión" gracias a la interpretación, ya que traducción e interpretación son quehaceres distintos. La traducción consiste en una actividad casi mecánica, donde una secuencia de palabras en un lenguaje es sustituida por otra secuencia en otro lenguaje, sin que haya pérdida de significado; esto es, se puede encontrar una relación isimórfica entre esos lenguajes. Por el contrario, en la interpretación no se utilizan dos lenguajes donde uno se "vacía" en el otro, sino que setiene el lenguaje original a interpretary es necesario aprenderlo, vivir en él y ver a través de él. Como lo señala Kuhn: "Cualquier cosa que se puede decir en un lenguaje puede, con suficiente imaginación y esfuerzo, ser comprendidapor un hablante de otro lenguaje. El requisito previo para tal comprensión, sin embargo, no es la traducción sino el aprendizaje del lenguaje."30 Pero la imposibilidad dela traducción "punto por punto" en algún momento nos llevará a encontrar en otro lenguaje algo que aunque se comprende no es posible traducirlo; así por ejemplo, "las personas bilingües reportan repetidamente que hay cosas que pueden expresar en uno de los lenguajes que no pueden expresar en otro." 31 Lo anterior simplemente significa que algo se entiende pero que no se puede hablar de él. ¿No cabría aquí recordar el aforismo de Wittgenstein que deloquenosepuredehablar es migr callar?32

La inconmensurabilidad también nos lleva a adoptar un concepto de verdad acorde con ese constructivismo internalista atenuado. D e ahí la afirmación de que el nuevo conocimiento reemplaza a otro distinto y no a la ignorancia o al error. Todo enunciado que está de acuerdo con el mundo creado, con la forma en que se percibe ese mundo, es verdadero; con la agravante de que esa forma es producto del enunciado. Así pues, los enunciados "el agua es un elemento" y "el agua está compuesta de hidrógeno y oxígeno"; "el sol gira alrededor de la tierra" y "la tierra gira alrededor del sol"; "los seres vivos no evolucionan" y "los seres vivos evolucionan"; "el flojisto es causa de la combustión" y "el oxígeno es causa de la combustión"; "los procesos de autoría, publicación y distribución de documentos son autónomos y claramente diferenciables en sus pasos y actores” y “los procesos de autoría, publicación

29 Ilid p. 231.

30 T. Kuhn. "D ubbing and Redubbing: The Vulnerability of Rigid D esignation". En W. Savage (ed), Saientific Theries Minnesta Studies in thePhilosqphy of Saiene Vo. XIV. Minneapolis: University of Minnesota Press, 1990. pp. 298-318. p. 300.

31 T. Kuhn. "Afterwords". En P. Horwich (ed) Wodd Changes Thomas Kuhn and theNature of Saience Cambridge, Massachusetts, 1993. pp. 311-341. p. 324.

32 L. Wittgenstein. Tradatus Lógị Filośíic 6.523. 
y distribución de documentos coinciden"; "la biblioteca es un espacio físico que posee colecciones organizadas y personal que da servicio a usuarios presenciales" y "la biblioteca no es un espacio físico y no posee colecciones organizadas aunque da servicios a usuarios a distancia", son verdaderos todos, cada uno en su matriz disciplinaria, sin que exista la posibilidad de contrastación entre ellos. Consideramos que negar un realismo externo, de un ser que, en palabras de Heidegger, se de-vele y des-cubra, no lleva a este tipo de relativismo epistémico.

Además, puesto que la elección entre paradigmas es una transición entre inconmensurables yno existe ni un mundo común, ni un lenguaje común, ni percepciones comunes, dicha elección no estájustificada ni por la lógica, ni por la experiencia neutral, ni por una prueba algorítmica. Por ello es necesario recurrir a la persuasión (eso sí "con buenas razones"), a la fe en el poder del nuevo paradigma, a la fidelidad profesional, eincluso ala autoridad extracientífica, para que tengalugar la "conversión" al nuevo paradigma. ${ }^{33}$ Pero siempre con la certeza un poco triste, como lo escribió Max Planck, de que "una nueva verdad científica no triunfa por medio del convencimiento de sus oponentes, haciéndoles ver la luz, sino más bien porque dichos oponentes llegan a morir y crece una nueva generación que se familiariza con ella."34

Consideramos que en el desarrollo de la ciencia en general y de la bibliotecología en particular la tradición tiene cabida, no como prejuicios dogmáticos aceptados por fe, autoridad o fidelidad y que pueden ser cambiados por otros gracias a una convesón, sino como premisas que permiten interrogar, interpretar e interpelar al objeto de estudio que ontológicamente existe en sí, aunque epistemológicamente haya sido construido (ipero siempre con base en su primacía ontológica!) y que tales premisas son aceptadas precisamente por aportary permitir respuestas sobre ese objeto de estudio. La inconmensurabilidad no es tal, la intraducibilidad total no existe gracias a ese mundo externo que posibilita el diálogo interparadigmático y no sólo intraparadigmático, que al final de cuentas es un monólogo.

Si existen la posibilidad dela comprensión, con todo lo que ello implica: laposibilidad de compartir sentidos y significados; la existencia del mundo externo y la verdad como la adecuación de los enunciados con ese mundo, entonces, a menos de correr el riesgo de recaer en el psicologismo ampliamente criticado por Husserl y desmentido por el desarrollo de la lógica en el siglo XX, eso muestrala posibilidad de la comunidad de comunidades, por lo menos en sentido epistémico y dialógico, porque se comparte un mundo ideal (sentidos y significados) en la comprensión y un mundo material con los referentes en el mundo externo. Si se "ve" otra cosa, como un tipo de nieve que los esquimales perciben claramentey nosotros no; o un gas que Lavosier llamó oxígeno y Priestley "aire común con una cantidad menor que la usual de flojisto"; 0 un objeto que algunos llaman "libro electrónico" y que para otros es todo "menos libro"; siempre es posible "ir a las cosas mismas" ideales (sentidos) o

33 T. Khun. La estructura delas revduaiones deetíficas México: FCE, 1996. pp. 234-246.

34 Max Planck. SaietificA utddiogaphyandOtherPapes pp. 33-34. Citado por Kuhn, T. Op Cit p. 235. 
materiales (referentes) para llegar a un acuerdo. Esto es posible, por supuesto, si se tiene la suficiente apertura para querer hacerlo y no negarse, como le sucedió a G alileo con sus oponentes, quienes tomaron la posición de "no querer mirar a través del telescopio." Pero ese acuerdo que se alcanza no es el resultado de un consenso o negociación, ni está basado en mi o nuestraverdad, sino en la verdad que corresponde a cierto análisis dela realidad. A hora bien, como la teoría es un sistema en donde existe una red de enunciados y conceptos que están interconectados y dependen y se modifican mutuamente, si dentro de ese sistema se introduce un nuevo concepto 0 enunciado que lo contenga y explique, se van a ver modificados todos los demás conceptos y enunciados. Pero esto significa que la tarea es clarificar y no negarse a aceptar nuevos conceptos. Se podría preguntar ¿y si los cambios son tan fuertes que se llega a un nuevo paradigma? Nuestra respuesta es que si esos cambios están de acuerdo con la realidad, nos ayudarán a profundizar en nuestro conocimiento pero no a romper con conocimientos anteriores que como tales están en concordancia con la realidad, aunque tal vez lo estén en otro nivel de profundización u otro enfoque, de ahí el paralelismo y la intraducibilidad "punto por punto" entre algunas teorías que parecen estudiar lo mismo.

Según nuestra opinión, uno de los problemas de las revoluciones científicas que se nos proporcionan como ejemplos es que nos muestran cambios de paradigmas erróneos a otros que no lo son (de la teoría del flojisto ala teoría de la combustión de Lavosier; de la astronomía de Aristóteles-Ptolomeo a la astronomía de GalileoNewton; de la teoría del creacionismo fijista a la teoría de la evolución) y por tanto, por supuesto, los primeros tienen que ser abandonados. ${ }^{35}$ ¿Pero qué sucede con avances sobre otros enfoques que no tienen que ser abandonados, por ejemplo con la física de Newton y la física de la relatividad, donde no se dan rompimientos y los físicos de todas las universidades se siguen educando en los dos paradigmas? De manera

35 Aunque estamos conscientes de que semejante afirmación en el mundo postmoderno actual puede parecer "la última pretensión del racionalismo y positivismo ya superados para alcanzar la verdad" y que incluso para el mismo Kuhn hablar de un paradigma erróneo y otro correcto carecería de sentido. No obstante considero que el realismo y el concepto de verdad como adecuación son los únicos instrumentos para huir del relativismo. Un ejemplo dramático que por cierto se rehuye (¿porque carece de importancia, por ignorancia o porque resulta incómodo?) es el cambio de paradigma propuesto en medicina, pero sin resonancia mundial en su momento, por el médico húngaro Ignaz Semmelweis, de cuando se desconocía e incluso se hacía burla de la asepsia, a cuando ésta se descubre y practica. A las mujeres que morían por fiebre puerperal por supuesto que no les sería indiferente el hecho de que hubieran podido haberse salvado si esa nueva idea (paradigma) hubiera triunfado en el pensamiento médico con anterioridad. ¿Puede haber algo más fuerte que la vida misma que rompa la inconmensurabilidad? Afirmar que los enunciados: "las causas de una infección se deben a la existencia de microorganismos que pueden ser eliminados y con ellos la infección misma" y "las causas de una infección no se deben a la supuesta existencia de microorganismos, por lo que es tan innecesario querer eliminarlos o no" son ambosvedadarosdependiendo del paradigma en que se afirmen, es algo que sólo puede ser expuesto en discusiones "filosóficas, especulativas", pero nos exponemos a recibir una bofetada si lo hacemos ante quienes sufrieron una pérdida por una infección que pudo haber sido evitada. 
semejante ¿un profesional de la información documental no estará obligado a estudiar los supuestos dos paradigmas, el tradicional que se centra en el mundo de los impresos, su organización y servicio en unidades informativas bien determinadas bibliotecas, archivos, y el nuevo, que estudia entes más abstractos como la información y el conocimiento y no sólo su organización sino su gestión, o fenómenos novedosos como documento electrónico o biblioteca virtual, entre otros? ¿No le ayudará ese estudio del primer paradigma para comprender y buscar las respuestas a las preguntas que le plantean las nuevas realidades? ¿No es el afán de realizar una ruptura con la tradición lo que obliga a "reinventar" de la nadalo que ya se tenía sobre descripción, organización y recuperación de documentos? ¿Por qué otros tienen que hacer tareas semejantes si ya existen profesionales que lo han venido haciendo durante años? ¿No se debe más bien a que quienes tienen el conocimiento (bibliotecólogos) no se han dado a la tarea de ponerlo en correspondencia con la realidad actual y a que quienes no tienen ese conocimiento (ingenieros y administradores principalmente) son quienes han tratado de hacerlo? ¿No es esto lo que ha ocasionado, en gran partela confusión, laintroducción de conceptos sin definiry de términos ambiguos? Nuestro diagnóstico es que si bien existe una realidad nueva que nos obliga a readaptar y desarrollar el conocimiento, aquello que nos proporciona el elemento de innovación, también es cierto que no se debe de partir de la nada, lo cual salva la tradición. La cuestión no es sustituir unos conocimientos por otros, sino profundizar, complementar, desarrollar unos conocimientos que se tienen y, sólo si es necesario, desechar aquellos que se creían tener pero que no eran tales. ${ }^{36}$

Una segunda propuesta que nos puede guiar para encontrar respuesta al dilema planteado inicialmente, sin caer en los inconvenientes que descubrimos en las ideas de las revoluciones científicas de Kuhn, es el planteamiento de otro filósofo de la ciencia Imre Lakatos.

\section{LOS PROGRAMAS DE IN VESTIGACIÓN CIENTÍFICA DE I. LAKATOS 37}

El modelo de Lakatos también va encaminado a reconstruir la historia de la ciencia y para ello introduce el concepto de programas de investigación científica. Para Lakatos el desarrollo de la ciencia no es un duelo entre dos: teoría (ciencia normal) y hechos (anomalías); sino entre tres: dos teorías en competencia y los hechos. Esas

36 De lo que se ha dicho anteriormente se puede deducir el concepto de conocimiento que manejamos: enunciado aceptado (creencia) verdadero y justificado. Dicho concepto es estrictamente considerado desde el punto de vista epistémico, lo que no significa que excluyamos la posibilidad de otros tipos de conocimiento. Este enfoque implica que si un enunciado que era aceptado y crédo por la mayoría resulta que no era verdadero, entonces eso no era conocimiento.

37 Los trabajos donde podemos encontrar las ideas de Lakatos sobre los programas de investigación científica son entre otros: "Falsación y la metodología de los programas de investigación científica" y "La historia de la ciencia y sus reconstrucciones racionales". A mbos reunidos en Imre Lakatos. La madoddoǵa delos programas deinvestigadóndientíica Madrid: Alianza Editorial, 1983. 
teorías surgen y se desarrollan dentro de un programa de investigación. A hora bien, el cambio de un programa de investigación científica a otro sucede, no como se da en Kuhn el cambio de paradigmas por una conversión casi mística gobernada más bien por la psicología social del conocimiento, sino por una evaluación racional con base en el dictamen de lo progresivo o degenerativo de los programas y no de una teoría aislada de ese programa. Cuando en una serie de teorías dentro de un programa se encuentran otras que logran realizar nuevas explicaciones y predicciones que sus predecesoras no hacían, el programa de investigación es teóricamente progresivo, y si además de eso dicho contenido teórico es confirmado, entonces el programa de investigación es empíricamente progresivo; en caso contrario se dice que es degenerativo. Una teoría puede ser reemplazada por otra que posee mayor contenido empírico confirmado, pero de ahí no se sigue que el programa anterior de investigación se abandone, sino más bien que gracias a que se encontró otra teoría, continúa vigente. El programa de investigación se cambia cuando todo él es degenerativo; esto es, cuando ya no se encuentran nuevas teorías con mayor contenido empírico que amplíen su alcance de explicación y predicción, además de que tengan en contra un programa rival que es progresivo.

Según Lakatos cada uno de los programas de investigación científica está constituido por tres elementos. El primero de ellos es el núcleo duro, que contiene las leyes, conceptos y supuestos fundamentales que le dan la característica definitoria al programa de investigación; gracias a él, el programa de investigación científica tiene una identidad propia. Este núcleo es infalseable por la decisión metodológica de los especialistas, es decir, no se puede atacar ni modificar, "está fuera de toda negociación". A sí por ejemplo, el núcleo duro de la física clásica está compuesto por las tres leyes del movimiento de Newton más su ley de gravitación universal; el núcleo duro de la astronomía de Copérnico-Galileo contienela tesis de que el sol estáinmóvil, de que los planetas, entre ellos la tierra, giran alrededor de él, y de que la tierra tiene un movimiento de rotación sobre su eje.

El segundo elemento del programa de investigación científica es el cinturón protector, formado por hipótesis auxiliares, condiciones iniciales, conceptos y terminología auxiliares, teorías, etcétera. Dicho cinturón se encuentra bajo la dirección que marca el núcleo duro y respetando los principios que éste le indica buscará ampliar las explicaciones de los fenómenos. A simismo, como su nombre lo indica, su función es proteger al núcleo duro del programa de posibles falseaciones. Todas las refutaciones serán recibidas y absorbidas por el cinturón protector, que es el que puede modificarse desechando, introduciendo o transformando algunos de sus elementos. Así por ejemplo, si el comportamiento de los planetas difería de lo predicho por la astronomía copernicana, se defendía el núcleo duro de ese programa añadiéndolo o modificando epiciclos a las órbitas de los planetas que en un principio se creían circulares; cambiando el cálculo de la distancia de la tierra a las estrellas; introduciendo nuevas condiciones iniciales; etcétera. 
El tercer componente del programa de investigación científica es la heurística, 0 conjunto de reglas metodológicas que indican lo que se debe hacer o está prohibido en el proceso de investigación. D e acuerdo con esto último la heurística puede ser negativa o positiva. La negativa nos indica lo que debemos evitar: atacar el núcleo duro; mientras que la positiva nos guía sobre los caminos que se deben seguir para resolver los problemas que se van presentando; es decir, nos indica cómo se ha de completar el núcleo duro para que pueda explicar los fenómenos, cómo cambiar y desarrollar las variables refutables, y cómo modificar el cinturón protector.

Esta concepción del desarrollo de la ciencia nos proporciona la posibilidad de conciliar los opuestos: innovación y tradición, ya que dentro de un mismo programa tienen cabida tanto la tradición dentro del núcleo duro, como la innovación dentro del cinturón protector.

Para cumplir con nuestrafinalidad de demostrar quelatradición einnovación tie nen lugar en el desarrollo de la ciencia bibliotecológica y de la información, proponemos una interpretación de las ideas de Lakatos. En primer lugar, consideramos que la inmunidad a la falseación del núcleo duro no es el resultado de un acuerdo metodológico entre los especialistas, sino la consecuencia que nos permite escuchar y comprender al ser que nos habla. El papel de la tradición como pre-juicio, como premisa para interpretar la realidad proclamado por G adamer, no implica ponerse, y no querer quitarse, unos anteojos de determinado color para ver la realidad de ese color, sino sólo tener un punto de apoyo, sacado de la realidad y para serle aplicado a la realidad, que sirva como principio de un camino interpretativo que nos conducirá una vez más a ese principio para reinterpretarlo, pero ahora desde un nuevo horizonte; esa tradición sólo sirve si permite ampliar el horizonte de investigación y valorar críticamente a la propia tradición. Los principios del núcleo duro de la $\mathrm{G}$ enética de Lysenko o de la Lógica "proletaria" (dialéctica) soviética, por ejemplo, no permitían la apertura de horizontes sino que determinaban su cerrazón; ni permitían la interpretación del fenómeno estudiado sino su velación, ymucho menos aún toleraban la valoración crítica de esos principios. D e ese modo no eran principios que le abrieran las puertas alainvestigación, sino cadenas que se lo impedían; por eso había que abandonarlos.

En segundo lugar proponemos una noción de programa de investigación científica más amplio aún que el sugerido por Lakatos. Así por ejemplo, tendríamos un programa de investigación en Biología cuya parte central (núcleo duro) sería la idea de la evolución, frente a otro programa cuyo núcleo duro sería el fijismo. D entro del primer programa se incluye la teoría de Lamarck, que explica el cambio morfológico por adaptación al medio ambiente basándose en la tesis fundamental de que la función crea al órgano y de que tales adaptaciones se transmiten hereditariamente, que posteriormente sería sustituida por la teoría de D arwin y su tesis de selección natural, y ésta a su vez sería complementada por la teoría integral de la evolución (neodarwinismo) que incluye además las ideas dela genética, la ecología y la biogeografía. 
Para Lakatos el programa de investigación sería la teoría de D arwin frente a la teoría de Lamarck, la cual no llegó a crear otras teorías.

En tercer lugar, otra aportación que le hacemos a Lakatos y que puede parecer contradictoria, es la idea de que el núcleo central puede ir modificándose sin que por ello cambie el programa de investigación. Esto sucede cuando se descubre que lo que se consideraba como el "corazón" de dicho programa en realidad no lo era, sino que a su vez se ha desprendido de otro núcleo más profundo pero no contradictorio con él, el cual determina no que se deseche el núcleo anterior sino su conservación, ahora como una teoría dentro del cinturón protector; y al mismo tiempo establece la aparición de otras teorías, cada una de ellas en un contexto determinado pero abarcado y contemplado por el nuevo núcleo central. Es esto lo que sucede con la teoría de D arwin y el neodarwinismo dentro del programa evolutivo, eincluso nos atreveríamos a decir, con las físicas clásica, de la relatividad y la cuántica, las cuales "trabajan" en un contexto epistemológico determinado, explicándolo cada una desde su "porción de realidad". Estas tres últimas quedan circunscritas dentro de un programa de investigación física que concibe el movimiento como desplazamiento de "cuerpos" en coordenadas espaciales, lo que excluye totalmente a la física aristotélica, que define al movimiento como cambio "cualitativo" en general de la "materia". Pero no se desechaa todas estas físicas, más bien coexisten, aunque no sean casos límite, generalizaciones o deducciones unas de otras. De ahí que sea posible responder ala pregunta planteada anteriormente, pues los biólogos y los físicos estudian en el primer caso a D arwin y al neodarwinismo, y en el segundo a las físicas de Newton, la de la relatividad y la cuántica, no cómo historia de la disciplina, sino como algo actual que todavía tiene vigencia.

\section{HACIA LA IDENTIFICACIÓN DEL NÚ CLEO DURO DE LA CIENCIA BIBLIOTE- COLÓGICA Y DE LA INFORMACIÓN}

Ese repensar y replantear los fundamentos dela cienciabibliotecológica es el camino que seguimos cuando hablamos de "mudarnos a una ontología superior," 38 lo cual consiste no en abandonar la tradición, realizar una ruptura y caer en una innovación absoluta, sino en buscar otro núcleo más abstracto y más profundo que permita el desarrollo dela disciplinay al mismo tiempo le dé cabidaa las teorías anteriores dentro del contexto de la realidad que les correspondeinvestigar, así como a otras teorías, también dentro de sus contextos novedosos, que sin embargo siguen teniendo algo de antiguo. ${ }^{39}$ Con esta aportación intentamos explicar el cambio y desarrollo ocurridos dentro de la ciencia

38 Miguel Ángel Rendón Rojas. Basesteéicasyfilosócicasdelabiblicteedoǵa UNAM/ CUIB, 1998. p. 112.

39 En el trabajo de la nota anterior hablamos de un cambio de paradigma. D espués de un análisis posterior nos dimos cuenta que ésa no era la terminología adecuada dadas todas las implicaciones kuhnianas que líneas arriba señalamos. Lo que queríamos decir era que debíamos abandonar la visión restringida de bibliotecología como mundo de bibliotecas, libros y administración, para adoptar directrices más abstractas pero conservando siempre la unidad. 
bibliotecológica. No es un cambio de un paradigma de la biblioteca a un paradigma de la información ni tampoco un reacomodo mecánico a la nueva realidad, sino el descubrimiento de que lo que se tenía como núcleo central no lo era en última instancia y que más bien se desprende de algo más profundo. Así, sin necesidad de desecharla, tal visión tradicional pasa al círculo exterior de las teorías donde continuará su función de explicar, predecir y comprender la parcela de la realidad que le corresponde y coexistirá con otras teorías que pueden o no sustituirla, si coinciden en el análisis de esa parcelay lo hacen progresivamente. D e este modo es posible una profundización y ampliación en el conocimiento del fenómeno documental.

En un principio se consideró que el núcleo duro de la bibliotecología era el mundo de los impresos, la biblioteca y las actividades que se realizaban dentro de ella para dar servicio a los usuarios, y sólo después se descubrió que ese núcleo no era tal sino que se desprendía de algo más profundo aunque no contradictorio con él. Por tanto esas teorías no se desecharon para dar paso a otro programa de investigación sino que se "mudaron" al cinturón protector para coexistir con otras teorías. Creemos que el de la economía es un caso similar cuando ésta descubre que la producción, distribución y consumo de bienes materiales ya no son aquello a lo que estaba "acostumbrada" que fueran, sino procesos que dentro de lo que ahora llaman sociedad de la información se ven afectados por los avances científico-tecnológicos y la gran cantidad de personas dedicadas a esa esfera; por el dinámico flujo de la información; por el crecimiento del área de servicios; por la globalización, etcétera. No obstante no por ello claman por un cambio de paradigma que olvide lo anterior. O tro ejemplo sería el de la biología, cuando en cierto momento ve aparecer objetos totalmente nuevos, como los virus; sin embargo esto no la hace olvidarse de la unidad biológica para intentar crear otra ciencia totalmente nueva.

El viejo problema filosófico de la unidad en la diversidad, se traslada a la unidad del fenómeno informativo documental en la diversidad. Si en el fenómeno documental encontramos información, objetos y sujetos, es posible que la unidad esté dada por uno de esos elementos, sin embargo encontramos que dicha unidad no la proporciona la información por sí misma, ya que requiere tanto del referente sobre el que habla como del sujeto que interprete esa información: no nos sirve un manual de operación de una máquina si no está esa máquina, o bien si está la información pero si nadie la lee; tampoco la unidad se puede encontrar en el objeto (referente) por sí mismo: de nada sirve que esté la máquina si no se tiene la información para operarla, ni el sujeto que la opere; eigualmente la unidad tampoco descansa en el sujeto: si no tiene ni máquina ni información, de nada sirve que esté ahí un sujeto. Más bien el fenómeno informativo documental encuentra su unidad en esos tres elementos conjuntamente. Esa unidad se puede descubrir en lo que he denominado Sistema de Información D ocumental (SID), que está constituido por los siguientes elementos: información, documento, institución informativa documental y usuario, así como por las interrelaciones que hay entre éstos cuando la finalidad es satisfacer las necesidades de información documental de un usuario a través de la activa 
participación de los elementos señalados. ${ }^{40}$ D icho sistema es el núcleo duro de la ciencia bibliotecológica y de la información, de él se desprenden otras relaciones y objetos (mundo ontológico), conceptualizaciones de los anteriores (generalizaciones semánticas), teorías para explicarlos, directrices para investigarlos, etcétera. Entre esas relaciones y objetos podemos mencionar las unidades de información, los fondos de información, las fuentes de información documental, y actividades tales como la representación del contenido, la descripción de forma y contenido, el análisis, la síntesis, la selección, la normalización, la organización y la sistematización de información y de documentos, los servicios y productos de información, la evaluación, la metría y la economía del flujo de información en su uso y generación, el proceso de creación del documento por su autor, el papel dela industria editorial, el diá logo (comunidad) entre lector-autor mediatizado por el documento, la relación información-sociedad, etcétera.

A hora sí podemos constatar que no importa si el fenómeno documental estudiado está en el contexto conventual de conservación, o en el contexto de la ilustración del servicio público, o en un contexto neoliberal de mercantilismo y poder, 0 dentro del contexto tecnológico de (perdón por el término) una gjberealidad 41 Tampoco importa si los soportes de lainformación cambian, o si se modifican los tipos 0 formas históricas quetoman los fenómenos documentales, incluso aquellos que aún no existen pero que en un futuro aparecerán; todos esos momentos hunden sus raíces en el SID entendido como nosotros lo proponemos: siempre habrá producción, organización, circulación y uso de información documental, así como usuarios, instituciones informativas documentales y documentos. Por supuesto que con cada contexto aparecerán nuevas relaciones, conceptualizaciones y teorías que explicarán esas nuevas formas de aparecer del ser del mundo documental y que no tenían lugar en otros contextos, pero la luchano es entre teorías, sino entre series de teorías generadas por un programa de investigación. ${ }^{42}$

40 El análisis y descripción del SID así como de sus elementos, se puede encontrar en Miguel Ángel Rendón Rojas. Op Cit Así como en el trabajo del mismo autor: "El papel del profesional de la información en el acceso y uso de la información" En: Lainfomadónend inidodelaeradetrónica: información, sociedadyteendoǵa. México: UNAM-CUIB; 1998. T. 2. pp. 241-271.

41 El mundo donde encontramos, entre otros fenómenos, lo que algunos han dado por llamar gybees pacio ghbecultura, ghberanthropos, inteligeniacoletiva, virtualidadde bibliotecas, documentos, pérdida de diferencias entre productor, editor y distribuidor de documentos. Dicho sea de paso, todos ellos términos muy ambiguos y que requieren de una debida conceptualización dentro de una teoría que explique esa realidad y que al mismo tiempo emana del nuevo núcleo central.

42 Una simple revisión de la literatura que se publica en bibliotecología, concretamente en el Centro Universitario de Investigaciones Bibliotecológicas, nos dará una muestra de la emergencia de estudios relacionados con nuevas realidades, pero que emanan, implícitamente, del núcleo de la disciplina. Algunos hablan de algo tan tradicional como el servicio de consulta: Richard Bopp y Linda C. Smith. Introdurcióngeneral al servidodeconsulta México: UNAM/ CUIB Fideicomiso para la Cultura México-EUA; Información Científica Internacional; Alfagrama, 2000. Pero en un capítulo se nos habla de "Los servicios electrónicos de consulta". pp. 73-100; y aún en otro de "Las oportunidades y los retos en las tendencias de los servicios electrónicos de consulta”. pp. 391-413. O tros más versan 
El programa de investigación que proponemos da lugar a la existencia de teorías, si alguien propone otro, tendrá que demostrar, primero, que no coincide en los presupuestos centrales con el nuestro, de lo contrario estaríamos hablando no de dos programas de investigación sino de uno; y segundo, si realmente es distinto, tendrá que dar cuenta (explicar, predeciry comprender) de todo el universo del mundo documental, con especialización de disciplinas, y sin olvidar, al mismo tiempo, la integración de la ciencia documental.

Asimismo ese núcleo central de la ciencia bibliotecológica y de la información será el que le proporcionará identidad propia a la disciplina y marcará sus fronteras, de tal forma que la ayude a diferenciarse de otras disciplinas. D e este modo, la ciencia bibliotecológica podrá relacionarse con otras áreas del saber, pero ya no desde una situación amorfa que por carecer de límites determinados invada terrenos que pertenecen a otras disciplinas o sea invadida por ellas, sino teniendo plena conciencia de su ser único e individual.

\section{CONCLUSIONES}

Podemos concluir el presente trabajo intentando responder la cuestión que guió nuestras reflexiones. El ulterior desarrollo de la cienciabibliotecológicay de la información no está supeditado a elegir entre su paradigma tradicional o el rompimiento con éste para crear uno nuevo. Esto significa que no es necesario quedarse únicamente en la tradición sin dar paso a la innovación, así como tampoco lanzarse a la innovación sin tomar en cuenta las raíces teóricas que nos comunican con el pasado. La propuesta es conciliar esos dos opuestos en una visión que refleje el desarrollo de la ciencia bibliotecológica, en la que, con la terminología de Lakatos, se recupere la tradición dentro de un núcleo central depurado que identificamos como el Sistema de Información D ocumental. Esenúcleo ha estado presente en todo el desarrollo de la disciplina aunque no se haya tenido conciencia de ello, porque en primera instancia aparecía otro núcleo identificado con el mundo fenoménico de las bibliotecas, los impresos y el servicio a los usuarios. Sólo después de la confrontación con nuevas realidades y el análisis de los fundamentos de la bibliotecología se descubrió la mayor profundidad del núcleo, y se advirtió que lo que anteriormente se tomaba como tal, emanaba realmente de un núcleo más abstracto y general.

sobre desarrollo de colecciones: Primer seminariointemadional sobredesamdlodecdeciones María del Carmen Negrete G utiérrez (Coord.) México: UNAM/ CUIB, 1998. Pero dentro de esa obra se escribe sobre "Criterios de selección y adquisición de materiales impresos y electrónicos" . pp. 135-173; y sobre "Propiedad de la información vs acceso remoto". pp. 175-202. Algunos más centran su atención en la catalogación pero de un nuevo tipo de documentos: Filiberto Martínez A rellano y Lourdes Milagros V allejo Hernández. Catalogadóndedbamentos detrónicos ardhivodedatosyprogramas México: Información Científica Internacional, 1998. Si continuáramos la enumeración, la lista sería muy extensa. 
Almismo tiempo se reconocelanecesidad de la innovación, la cual toma lugar en el cinturón protector de la disciplina; es ahí donde se dan las transformaciones, modificaciones o eliminaciones en las construcciones teóricas. D entro de este cinturón protector encontramos una serie de teorías novedosas que surgen del nuevo núcleo, dentro del cual cada una de ellas investiga en su contexto determinado. La única condición que tales teorías deben satisfacer es que amplíen su contenido teórico; esto es, que su campo de explicación y predicción sea cada vez mayor y que los hechos empíricos confirmen ese contenido teórico, lo que significará que su carácter es progresivo. 